\title{
Increased Ocular Pulse Amplitude Associated with Unilateral Dysgenesis of the Orbital Roof
}

\author{
Ami Shah Vira ${ }^{a} \quad$ Ashraf M. Mahmoud ${ }^{a, b} \quad$ Cynthia J. Roberts ${ }^{a, b}$ \\ Steven E. Katz ${ }^{a, b}$ \\ Departments of ${ }^{a}$ Ophthalmology and ${ }^{b}$ Biomedical Engineering, The Ohio State University, \\ Columbus, Ohio, USA
}

\section{Key Words}

Ocular pulse amplitude Cerebrospinal fluid pulse $\cdot$ Sphenoid wing dysplasia $\cdot$ Orbital roof dysgenesis · Intracranial pressure

\begin{abstract}
Introduction: Two patients (one with neurofibromatosis type 1) presented with unilateral ocular pulsation. Methods: A CT scan of the orbits revealed extensive dysgenesis of the orbital roof with herniation of the frontal lobe into the orbit in both cases. PASCAL dynamic contour tonometry was performed. Results: The ipsilateral ocular pulse amplitude (OPA) was greater than the contralateral side, and the ocular pulse waveform morphology more closely approximated the known intracranial waveform in these patients. Conclusions: We hypothesize that the greater OPA was due to stronger transmission of the intracranial pressure waveform amplitude and morphology in the absence of the orbital roof.

(C) 2015 S. Karger AG, Basel
\end{abstract}

\section{Introduction}

Intraocular pressure (IOP) is pulsatile due to the interdependence of ocular blood flow and the aqueous circulation [1] and can reflect systemic hemodynamic events [2]. In addition, the amplitude of the pulsations may be affected by high intracranial pressures (ICPs) [3, 4]. Ocular pulse amplitude (OPA) is defined as the difference between systolic and diastolic IOP. Average values of OPA in healthy subjects range from 1 to $4 \mathrm{~mm} \mathrm{Hg}$. The purpose of this report was to demonstrate the source for asymmetric OPAs and waveform morphologies 
Vira et al.: Increased Ocular Pulse Amplitude Associated with Unilateral Dysgenesis of the Orbital Roof

between fellow eyes of 2 subjects with extensive bony orbital roof defects, allowing stronger transmission of the ICP waveform morphology to the ocular pulse waveform.

\section{Case Reports}

Case 1

A 55-year-old white male with a known history of neurofibromatosis type 1 (NF-1) presented with chief complaints of pulsation of the right eye affecting his vision and a progressive tumor involving the right upper eyelid over several years. An infiltrative tumor was noted involving the right upper eyelid, causing mechanical ptosis and extending into the anterior orbit (fig. 1a). Exophthalmometry measurements were $9 \mathrm{~mm}$ OD and $17 \mathrm{~mm}$ OS at a base of $96 \mathrm{~mm}$. Visual acuities with present glasses were 20/100 OD and 20/20 OS with no relative afferent pupillary defect. Slit lamp evaluation was significant for Lisch nodules and early nuclear sclerotic cataracts bilaterally. IOPs were 15-19 mm Hg OD and 15-16 mm Hg OS by Goldmann applanation tonometry, with the mires on the right displaying greater pulsations. Funduscopic evaluation revealed cup-to-disc ratios of $0.4 \mathrm{OD}$ and $0.5 \mathrm{OS}$, and the maculae, vessels and peripheries were within normal limits. There was decreased infraduction OD, a right hypertropia of $40 \mathrm{dpt}$ and exotropia of $20 \mathrm{dpt}$ in primary gaze. CT scan of the orbits showed the extent of the upper eyelid tumor on the right and also revealed absence of the sphenoid wing including the lateral orbital roof and most of the lateral wall on the right (fig. 1b). Anterior orbitotomy was performed via an upper eyelid crease incision for excisional biopsy of the tumor. Surgical pathology was consistent with plexiform neurofibroma. PASCAL dynamic contour tonometry average IOPs (performed preoperatively) were 18.5 $\mathrm{mm} \mathrm{Hg} \mathrm{OD} \mathrm{and} 16.8 \mathrm{~mm} \mathrm{Hg}$ OS with OPAs of $2.87 \mathrm{~mm} \mathrm{Hg}$ OD and $1.47 \mathrm{~mm} \mathrm{Hg}$ OS, which was 16 and $9 \%$ of IOP, respectively (fig. 1c).

Case 2

A 55-year-old white female with no stigmata of NF-1 presented with a chief complaint of pulsation of the right eye slowly progressing in the past 2 years (see online suppl. video 1; for all online suppl. material, see www.karger.com/doi/10.1159/000430925). Visual acuities without correction were $20 / 25$ OU with no relative afferent pupillary defect. Slight lateral and inferior displacement of the right globe was noted; however, there was no axial displacement noted on exophthalmometry (fig. 2a). Ductions were noted to be full with no evidence of ocular misalignment. Slit lamp evaluation was unremarkable with the exception of early nuclear sclerotic cataracts bilaterally. No Lisch nodules were noted. IOPs were 10$11 \mathrm{~mm} \mathrm{Hg}$ OU by Goldmann applanation tonometry, with the mires on the right displaying greater pulsations. Funduscopic evaluation revealed cup-to-disc ratios of $0.15 \mathrm{OU}$, and the maculae, vessels and peripheries were within normal limits. A CT scan of the orbits showed a large bony defect involving the right orbital roof, including the frontal bone as well as the greater and lesser wings of the sphenoid bone. Herniation of the right frontal lobe into the superomedial orbit was noted (fig. 2b). PASCAL dynamic contour tonometry average IOPs

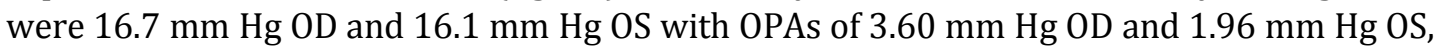
which was 22 and $12 \%$ of IOP, respectively. 
Vira et al.: Increased Ocular Pulse Amplitude Associated with Unilateral Dysgenesis of the Orbital Roof

\section{Discussion}

NF-1 is a condition that affects the skin, peripheral nervous system and skeletal system. It results from defects on chromosome 17, which either arise as mutations or are inherited as autosomal dominant with an incidence of 1 in 3,000 live births $[5,6]$. The diagnostic criteria were set forth by the National Institute of Health Consensus Development Conference in 1988 [7]. The most common clinical hallmark of ocular involvement of neurofibromatosis is iris Lisch nodules followed by choroidal hamartomas.

Patients with NF-1 may present with osseous dysplasia of the orbit, usually with hypoplasia of the greater sphenoid wing. The osseous dysplasia is thought to be due to associated mesodermal developmental abnormalities rather than direct relation to eroding neurofibromas. Theories into the cause of sphenoid wing dysplasia (SWD) include: being part of a congenital anomaly, maldevelopment of the centers of ossification, an increase in overall cerebral volume leading to bony changes and abnormal vascularity contributing to bone resorption adjacent to the superior orbital fissure [8].

The incidence of SWD ranges from 4 to 58\% [5]. SWD may result in exophthalmos and/or downward displacement of the globe associated with brain herniation into the orbit. Alternatively, SWD may be associated with relative enophthalmos due to orbital expansion or fat atrophy. Sphenoid dysplasia is usually unilateral and occurs more commonly on the left than right. Increased ocular pulsation may occur from transmission of intracranial pulsations to the orbit through the bony defect. The association of SWD with pulsating exophthalmos was first described by Dandy in 1929 [9].

Absence of a portion of the orbital roof may also be seen with trauma including orbital roof fractures and after surgical excision of tumors involving the greater sphenoid wing, such as meningiomas. Our case 2 had no history of trauma or intracranial surgery; therefore, it seems likely that this was an isolated case of dysgenesis with thin bone that gradually eroded secondary to repeated insult of the cranial pulse.

In normal anatomy, the cerebrospinal fluid (CSF) pulse is transmitted through the orbital tissues via the small area surrounding the optic nerve where CSF pressure (CSFP) directly impinges on the peripapillary structures. However, when a significant portion of the orbital roof and lateral wall are absent, the CSF pulse is directly transmitted to the orbital tissues over a much larger area and magnifies the contribution of the CSFP to the OPA. We described 2 patients with substantial bony defects of the orbital roof, including one with NF1 , SWD, symptomatic pulsatile enophthalmos and an elevated OPA on the affected side. We hypothesize that the greater OPA was due to stronger transmission of the ICP waveform amplitude and morphology in the absence of the separation usually supplied by the sphenoid wing (fig. 1d). In the 2 nd subject, a large bony defect produced similar asymmetry in the OPA, with the affected side having a larger magnitude due to greater surface area of CSFP directly transmitted to the eye. It is possible to repair the bony defect via a pterional craniotomy with a split thickness calvarial graft or titanium mesh [10]. Presumably, repair of the bony defect would normalize the OPA compared to the contralateral side by significantly reducing the mechanical effect of the ICP pulse.

\section{Statement of Ethics}

Both patients have consented to the submission of this case report for publication. One or two case reports [involving description of clinical features and/or outcome of case(s) without additional evaluation, analysis, or review of others for comparison] does not consti- 
Vira et al.: Increased Ocular Pulse Amplitude Associated with Unilateral Dysgenesis of the Orbital Roof

tute research involving human subjects, and therefore does not require IRB review or exemption per our institutional policy.

\section{Disclosure Statement}

Steven E. Katz, Ami Shah Vira, and Ashraf M. Mahmoud certify that they have no affiliations with or involvement in any organization or entity with any financial interest (such as honoraria; educational grants; participation in speakers' bureaus; membership, employment, consultancies, stock ownership, or other equity interest, and expert testimony or patent-licensing arrangements) or nonfinancial interest (such as personal or professional relationships, affiliations, knowledge or beliefs) in the subject matter or materials discussed in this article. Cynthia J. Roberts is a consultant for Ziemer Ophthalmic Systems, manufacturer of the PASCAL dynamic contour tonometer.

\section{References}

1 Karol HJ, Roberts CJ, Small RH: Electrical analog model of ocular pulse amplitude as a function of systemic pulse pressure and ocular rigidity. Invest Ophthalmol Vis Sci 2007;48:ARVO E-Abstract 4946.

-2 Kassem JB, Katz SE, Small RH, Raman SV, Roberts CJ: Ocular pulse amplitude waveform reflects ventricular bigeminy and aortic insufficiency. Indian J Ophthalmol 2015;63:59-61.

3 Bolisetty K, Roberts CJ, Mahmoud AM, Okon M, Katz SE: Correlation of change in ocular pulse amplitude with change in intracranial pressure after lumbar puncture. Invest Ophthalmol Vis Sci 2013;54:ARVO EAbstract 4366.

4 Katz SE, Mahmoud AM, Okon M, Bolisetty K, Small RH, Roberts CJ: Changes in intracranial pressure (ICP) and ocular pulse amplitude (OPA) in patients with idiopathic intracranial hypertension (IIH). Invest Ophthalmol Vis Sci 2013;54:ARVO E-Abstract 4367.

5 Farris S, Grove A: Orbital and eyelid manifestations of neurofibromatosis: a clinical study and literature review. Ophthalmic Plast Reconstr Surg 1996;12:249-259.

6 Friedman JM: Epidemiology of neurofibromatosis type 1. Am J Med Genet 1999;89:1-6.

7 Neurofibromatosis. Conference statement. National Institutes of Health Consensus Development Conference. Arch Neurol 1988;45:575-578.

8 Macfarlane R, Levin A, Weksberg R, Blaser S, Rutka J: Absence of the greater sphenoid wing in neurofibromatosis type I: congenital or acquired: case report. Neurosurgery 1995;37:129-133.

-9 Dandy WE: An operative treatment for certain cases of meningocele (or encephalocele) into the orbit. Arch Ophthalmol 1929;2:123-132.

10 Lofty M, Xu R, McGirt M, Sakr S, Ayoub B, Bydon A: Reconstruction of skull base defects in sphenoid wing dysplasia associated with neurofibromatosis 1 with titanium mesh. Clin Neurol Neurosurg 2010;112:909914. 


\section{Case Reports in \\ Ophthalmology}

Vira et al.: Increased Ocular Pulse Amplitude Associated with Unilateral Dysgenesis of the Orbital Roof
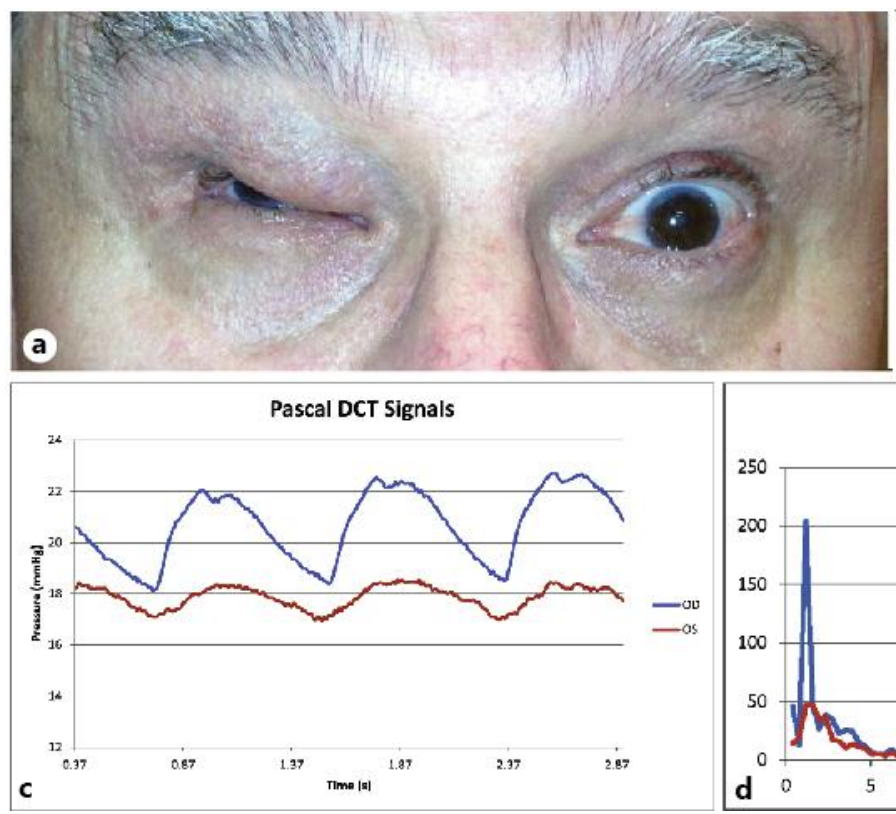

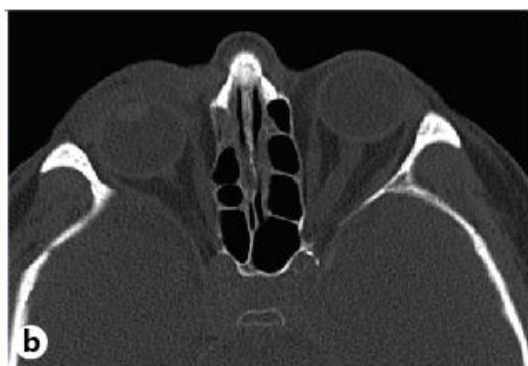

Frequency Spectrum

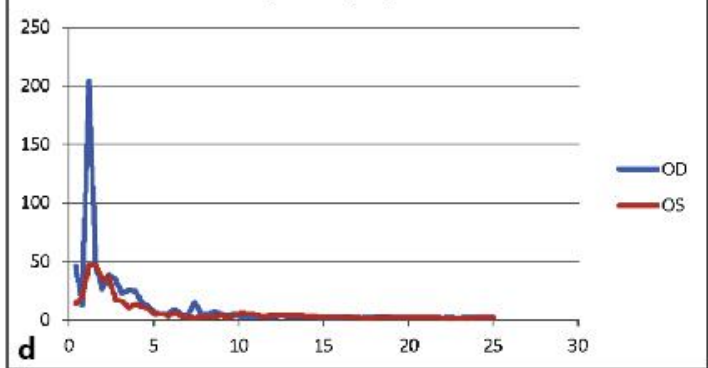

Fig. 1. a Case 1, a 55-year-old male presenting with progressive soft tissue mass in the right upper eyelid associated with notable pulsating right enophthalmos. b CT scan, bone window, showing absence of the right sphenoid wing and right relative enophthalmos. c Average IOP and OPA OD: 18.5 (range 18.3-18.8) and 2.87 (range 2.2-3.5) mm Hg. Average IOP and OPA OS: 16.8 (range 16.6-16.9) and 1.47 (range 1.41.6) $\mathrm{mm} \mathrm{Hg}$. The depicted waveforms have IOP and OPA of OD: 18.8 and $3.5 \mathrm{~mm} \mathrm{Hg}$, and OS: 16.8 and 1.4 $\mathrm{mm} \mathrm{Hg}$. The eye with absence of the sphenoid wing of the orbit (OD) has not only greater OPA, but also has a waveform morphology that is more similar to that of ICP with multiple peaks. $\mathbf{d}$ Fast Fourier transform of waveforms in figure 2a showing frequency content of OD waveform with lack of corresponding peaks in OS waveform. This demonstrates the distinct waveform morphology between the eyes, in addition to the difference in waveform amplitude. 
Case Reports in

Ophthalmology

Vira et al.: Increased Ocular Pulse Amplitude Associated with Unilateral Dysgenesis of the Orbital Roof
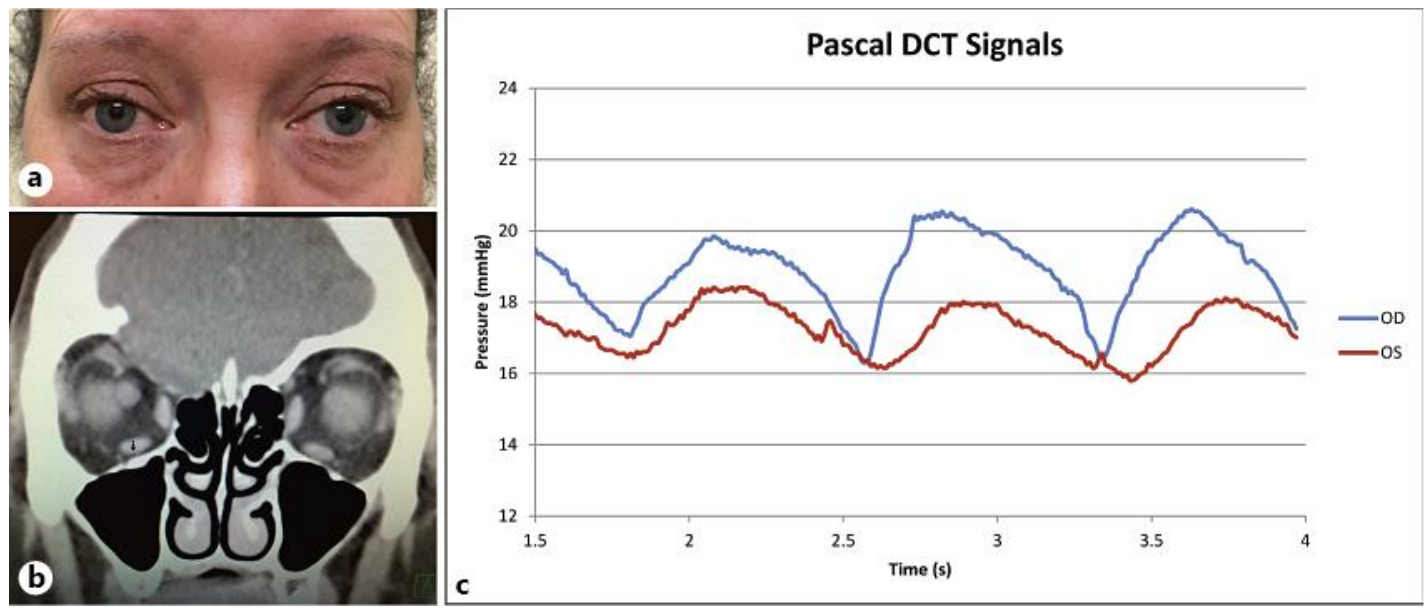

Fig. 2. a Case 2, a 55-year-old female presenting with right ocular pulsation associated with slight downward and lateral globe displacement. b Coronal CT scan showing absence of the right superomedial orbital roof with herniation of the frontal lobe into the orbit. c Average IOP and OPA OD: 16.7 (range 16.4-16.9) and 3.60 (range 3.5-3.7) mm Hg. Average IOP and OPA OS: 16.1 (range 15.9-16.5) and 1.97 (range 1.72.1) $\mathrm{mm} \mathrm{Hg}$. The depicted waveforms have IOP and OPA of OD: 16.9 and $3.5 \mathrm{~mm} \mathrm{Hg}$, and OS: 16.4 and 2.1 $\mathrm{mm} \mathrm{Hg}$. Once again, the eye with dysgenesis of the orbital roof (OD) has elevated OPA as well as a waveform morphology that more closely approximates the intracranial pulse. 